\title{
Ice skating promotes postural control in children
}

\author{
M. Keller ${ }^{1}$, K. Röttger², W. Taube ${ }^{1}$ \\ ${ }^{1}$ Movement and Sport Science, Department of Medicine, University of Fribourg, Fribourg, Switzerland, ${ }^{2}$ Department of Sport and \\ Sport Science, University of Freiburg, Freiburg, Germany \\ Corresponding author: Martin Keller, Movement and Sport Science, Department of Medicine, University of Fribourg, Boulevard de \\ Pérolles 90, CH-1700 Fribourg, Switzerland. Tel: +41 2630072 86, Fax: +41 2630097 71, E-mail: martin.keller@unifr.ch
}

\begin{abstract}
High fall rates causing injury and enormous financial costs are reported for children. However, only few studies investigated the effects of balance training in children and these studies did not find enhanced balance performance in postural (transfer) tests. Consequently, it was previously speculated that classical balance training might not be stimulating enough for children to adequately perform these exercises. Therefore, the aim of this study is to evaluate the influence of ice skating as an alternative form of balance training. Volunteers of an intervention $(n=17$; INT: $13.1 \pm 0.4$ years $)$ and a control group $(n=13$; CON: $13.2 \pm 0.3$ years) were tested before and after training in
\end{abstract}

static and dynamic postural transfer tests. INT participated in eight sessions of ice skating during education lessons, whereas $\mathrm{CON}$ participated in normal physical education. Enhanced balance performance was observed in INT but not in CON when tested on an unstable free-swinging platform $(P<0.05)$ or when performing a functional reach test $(P<0.001)$. This is the first study showing significantly enhanced balance performance after ice skating in children. More importantly, participating children improved static and dynamic balance control in postural tasks that were not part of the training.
An intact balance performance constitutes the basis for almost all daily movements such as walking, grasping, or reaching and is essential in the motor development of children. The ability to control static and dynamic balance is important and necessary for participation in activities of daily living and physical activity (De Oreo $\&$ Keogh, 1980). However, it is well known that young children (Riach \& Hayes, 1987) as well as elderly people (e.g., Baudry \& Duchateau, 2012) use different postural control strategies to those of healthy young adults, and that the weighing of sensory information alters between different age groups. Interestingly, these two age groups are also at higher risk of falling (Granacher et al., 2011a). The financial costs caused by falls are extremely high in the elderly (Stevens et al., 2006), but - and this may surprise - are also very high in children (Mathers \& Penm, 1999). Mathers and Penm (1999) estimated the direct costs of falls in children for the Australian healthcare system to be $\$ 133.8$ million per year. This means that the Australian health-care system is charged by around $\$ 35$ per child and year due to the medical treatment of falls. This is double the amount that is spent for people aged 25-44 years (Mathers \& Penm, 1999). Furthermore, another study assessed age- and genderspecific injuries and tried to identify risk groups and risk factors for injury prevention (Kahl et al., 2007). The authors found that $15.2 \%$ of the children (aged 1-17 years) had at least one accident in the year before the survey. Interestingly, injuries in the observed cohort were mainly caused by falls on level ground (35.2\%), falls from heights $(25.2 \%)$, and collisions with objects or persons $(20.6 \%)$. Thus, the most frequent injury mechanisms were all related to postural control and/or locomotor tasks. For the United States and Germany, it is reported that falls are the most common cause of pediatric injury and also the leading cause of injury transported to emergency departments (Kahl et al., 2007; Owens et al., 2008).

Based on the increasingly sedentary behavior and the associated decline of motor abilities of children observed over the last decades (Jurimae et al., 2007; Bös et al., 2008), the problem of falls and fall-related injuries may become even greater in the future. To counteract this development, it seems plausible to design adequate training programs to improve sports-related performance in general and postural skills in particular to reduce intrinsic risk factors of falls like deficits in static and dynamic balance or strength (in line with propositions made by Granacher et al., 2011a). This may not only reduce the financial burdens to the health-care system, but also, more importantly, the incidence of falls and fall-related injuries in children.

Numerous studies investigated the effects on postural control of classical balance training incorporating instable devices such as half boards, spinning tops, soft mats, etc. known from physiotherapy (Gruber \& 
Gollhofer, 2004; Taube et al., 2007b; Granacher et al., 2011b). In adults, these training interventions did not only improve postural control but also showed numerous "side effects." For example, classical balance training also enhanced the rate of force development and/or jump height (Gruber \& Gollhofer, 2004; Taube et al., 2007b), and its application reduced the incidence of non-contact sports injuries (for an overview, see Taube et al., 2008). Apart from classical balance training, alternative approaches such as skiing (Lauber et al., 2011) or slacklining (Keller et al., 2012; Pfusterschmied et al., 2013) were shown to improve postural control in adults. Thus, the different beneficial effects of balance training have been investigated manifold times for adults and elderly people. However, only a few studies scrutinized the impact of balance training on stance stability in children. Ledebt et al. (2005) were the first authors to investigate the effects of balance training with visual feedback on stance and gait parameters in children suffering from hemiplegic cerebral palsy. The authors observed improvements both in gait and stance in their subjects. Similar observations were made by Mombarg et al. (2013) investigating the effects of a 6-week Wii intervention on balance performance in children with poor motor performance. The authors described significantly improved balance scores in the training group without any changes in the control group. One can question, however, whether the results of patients and subjects with poor motor performance can be transferred without any limitations to healthy children. Hence, Granacher et al. (2011b) examined for the first time whether 4 weeks of classical balance training improves postural control in healthy pre-pubertal children. Surprisingly, the authors were not able to find any significant interaction effects between groups and time and observed only small to medium effect sizes, although a training program was used that proved to be efficient in young adults (Taube et al., 2007a) and old subjects (Granacher et al., 2010c). Importantly, it was mentioned that high levels of attentional focus and concentration were only achieved by the children when coaches were standing right beside them (Granacher et al., 2011b). This might have been due to the young age and immaturity of the subjects. Thus, one may speculate that classical balance training might not be suitable to improve balance in children as the executed tasks (balancing on air cushions, half boards, etc.) might be of little interest to them. Consequently, it seems reasonable to use innovative devices for training children that are more appealing and motivating. A recent study therefore tested whether slackline training improves postural control in children (Donath et al., 2013). After the training, the authors observed significantly enhanced performance when balancing single- and double-legged on the slackline, but they did not find any significant transfer effect when testing static and dynamic single limb stance or backward balance beam walking. In line with this observation, there is one slackline training study in young adults also showing no transfer to other balance tests (Granacher et al., 2010b). Thus, although there are two further studies in young adults demonstrating increased postural stability in transfer tasks (Keller et al., 2012; Pfusterschmied et al., 2013) it might nevertheless be speculated that the adaptations in response to slackline training are very task specific and probably not as broad as the ones reported after conventional balance training where different devices demand joint stability in all directions.

Therefore, the aim of the current study was to investigate further the effects of an innovative training regimen on balance performance in children. The participating subjects of the training group conducted a 4-week ice skating training regimen consisting of ice skating with additional tasks to keep the subjects alert and motivated during all training sessions. Because of the high stimulative nature of ice skating and studies showing improved balance performance after inline skating in the elderly (Taube et al., 2010), we expected an enhanced postural control after the training program in the intervention but not in the control group.

\section{Methods}

To evaluate the effects of a 4-week ice skating training regimen on postural control, we measured the subjects from an intervention group (INT) and a control group (CON) before and after the training. All tests were approved by the local ethics commission, and all measurements were conducted in accordance with the latest version of the Declaration of Helsinki and the Ethical Standards in Sport and Exercise Science Research (Harriss \& Atkinson, 2011)

\section{Subjects}

For the present study, we provided verbal and written information to the parents and children before the start of the study. Parents gave written informed consent before the children were included. All children were free of orthopedic and/or neurological disorders that might have affected the outcome of the study. None of the children had participated in systematic balance and/or resistance training and none had participated in any ice skating training prior to the start of the current study. Overall, 33 schoolchildren were included and were allocated to either INT or CON. Three subjects of CON dropped out of the analysis as they did not participate in the post-test due to illness. Hence, 17 subjects (boys: 9; girls: 8; age: $13.1 \pm 0.4$ years, size: $155.8 \pm 7.6 \mathrm{~cm}$; weight: $45.2 \pm 8.6 \mathrm{~kg}$ ) in the INT and 13 subjects (CON: boys: 7; girls: 6; age: $13.2 \pm 0.3$ years, size: $156.1 \pm 8.2 \mathrm{~cm}$; weight: $46.1 \pm 6.7 \mathrm{~kg}$ ) in the CON were used for final analysis. All subjects were recruited from the same school.

\section{Training}

The training paradigm lasted for 4 weeks and consisted of two sessions per week. Each session lasted for $90 \mathrm{~min}$. The main aspects of the training sessions are summarized in Table 1. Care was taken that the difficulty of the exercises was increased throughout the training regimen. All subjects wore helmets and protective equipment such as wrist and knee guards. All female 
Table 1. The postural demands were enhanced with improvements in ice skating to meet the actual skill level of the subjects

\begin{tabular}{ll}
\hline Week & Main aspects of the training sessions \\
\hline 1 & Standing and walking on a carpet \\
& How to fall properly \\
& Basic skating technique \\
& Skating in a special course requiring different postures \\
& Skating with dual tasks (catching a ball or dribbling) \\
& Special techniques for curves (leaning inwards) \\
3 & Basic technique for skating backwards \\
& Jumps and landings \\
& How to hockey stop \\
& Different games: playing tag, shuttle races, etc. \\
& Basic techniques of ice hockey (boys) \\
& Basic techniques of figure skating (girls) \\
\end{tabular}

The main aspects of the training sessions are summarized in this table.

participants wore figure skates, whereas all male volunteers were equipped with ice hockey skates. We chose a local indoor ice rink as training ground in order to have similar training conditions independently of the weather. The training was held during normal physical education classes: subjects either participated in the normal physical education (CON) or in a special ice skating training supervised by two coaches experienced in ice hockey and/or figure skating (INT). Therefore, the amount of physical education was identical for both groups, but different with respect to the taught content (ice skating vs normal physical education such as volleyball and gymnastics). We chose this paradigm in order to have a similarly active CON group and not to add any physical activity for the INT group only.

\section{Measurements conducted in the pre- and post-test}

For the evaluation of postural control, a balance device called Posturomed $^{\mathrm{TM}}$ (Haider Bioswing, Pullenreuth, Germany) was used. The multi-axial free-swinging support platform is mounted on four pendulums to guarantee free movements in all directions in the transversal plane. A custom-built joystick potentiometer was connected to the platform for the evaluation of the sway path of the platform in antero-posterior and medio-lateral $(\mathrm{ml})$ direction. The same device was used in different recent publications (Taube et al., 2010; Keller et al., 2012; Pfusterschmied et al., 2013) and was shown to provide good test-retest reliability for different age groups (Boeer et al., 2010). It has to be mentioned, however, that none of the age groups evaluated by Boeer et al. (2010) corresponded to the age group used in the present study. Thus, the shown test-retest reliability cannot be transferred to our age group without limitations. All subjects were instructed to minimize the movements of the platform as much as possible. As subjects can adapt very rapidly to the characteristics of the device at the beginning of exposure, a period of familiarization was allowed. This period included 2 min of single-leg standing without perturbation and of five perturbations in $\mathrm{ml}$ direction in order to minimize the learning effects during data acquisition. During all measurements, subjects were instructed to stand with their supporting leg slightly bent $\left(20-30^{\circ}\right)$ and with hands on the hips. Postural control was tested in two different ways during right-legged stance. First, during unperturbed upright stance subjects stood on the freeswinging platform and were asked to minimize the movements of the platform during a period of $30 \mathrm{~s}$. Subjects repeated this task three times and the best trial was used for later analysis (Granacher et al., 2010b). Second, the sway of the platform was evaluated after a perturbation. In this lateral perturbation task, the platform was deflected $2.5 \mathrm{~cm}$ in the $\mathrm{ml}$ direction and fixed with an electromagnet. As long as the magnet was switched on, the platform was kept in the deflected position and the subjects could stand on it comparable with standing on solid ground. However, switching off the magnet resulted in an acceleration of the platform experienced as an ml perturbation (for technical details, see Muller et al., 2004). Subjects were asked to minimize platform oscillations during a period of $10 \mathrm{~s}$ after the perturbation that occurred with random delay after subjects stood in the starting position on the device. Again, the children were asked to repeat the measurements three times and the best trial was used for later analysis. There are clear recommendations provided how to maximize the reliability of center of pressure measurements (Ruhe et al., 2010), but these recommendations cannot be transferred to the device used in the present study. Thus, the used durations are based on our experience with the Posturomed and aimed to avoid fatigue.

A functional reach test (FRT) was conducted to measure the maximal forward reach before and after the training. This test was argued to be a simple but precise and reliable measure of balance (Duncan et al., 1990). Therefore, the subjects were asked to perform slow maximal forward reaches without losing contact of the heels. The maximum forward reach was measured using a custom-built device. The device consisted of a solid rail that was mounted to the wall in horizontal direction. Subjects could push a slider away from its starting position by reaching and leaning forward. A lever was mounted to the slider in vertical direction to guarantee that - independently of the body height subjects could always touch the lever and move it forward. A measuring tape was used to measure the final position of the slider that represented the reaching distance of the subjects. This implies that the larger the distance, the better the performance.

\section{Data treatment and statistical analysis}

To identify potential differences between the two groups in the pre-test, one-way analyses of variance (ANOVAs) were calculated. To evaluate differences in balance performance after the training a repeated-measures ANOVA procedure was calculated with the between-subject parameter group (INT vs CON) and the within-subject parameter time (pre vs post) and task (unpertubed upright stance condition vs the lateral perturbation task) [2 (group) $\times 2$ (time) $\times 2$ (task)]. Differences in the FRT test were evaluated using a repeated-measures ANOVA with the betweensubject parameter group (INT vs CON) and the within-subject parameter time (pre vs post) [2 (group) $\times 2$ (time) $]$. In case of significant $F$-values $(P<0.05)$, specific differences were investigated using Bonferroni corrected paired two-sided Student's $t$-tests.

\section{Results}

\section{Adaptations in postural control}

Groups did not show different performances before training (all values: $P>0.3$ ). However, the ice skating training resulted in significantly different adaptations between groups over time with significant improvements in the INT group, whereas the CON group did not show any significant adaptations (time $\times$ group interaction: $F_{1 ; 28}=5.37 ; P=0.028$; for details, see Figs. 1 and 2). Furthermore, the two groups differed significantly with respect to their changes in FRT from pre-test to postmeasurement (time $\times$ group interaction: $F_{1 ; 28}=14.96$; $P<0.001$; Fig. 3), meaning that only subjects of INT showed a significantly increased reaching distance. 


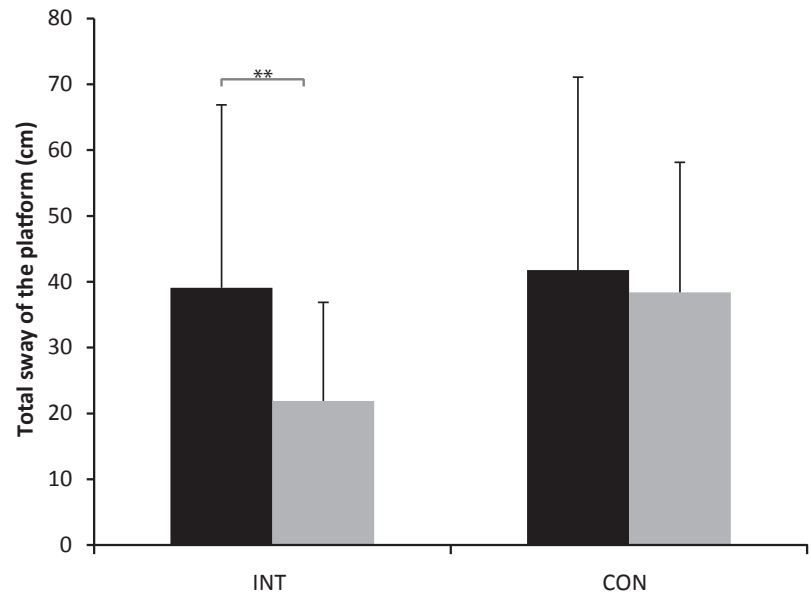

Fig. 1. In the unperturbed upright stance condition, the performance of the groups differed (time $\times$ group interaction) after training. The intervention (INT) group showed a highly significant improvement of performance (less platform movements), whereas the performance of the control $(\mathrm{CON})$ was unaltered $(* * P<0.01)$.

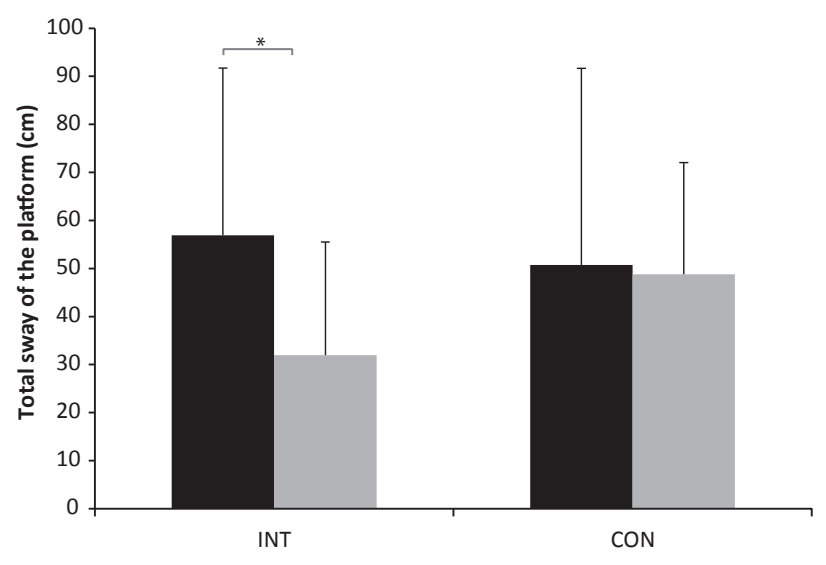

Fig. 2. A significant time $\times$ group interaction was found after the training period for the lateral perturbation task. The intervention (INT) group enhanced performance due to the participation in the training regime, whereas the control $(\mathrm{CON})$ group did not show any alterations $(* * P<0.05)$.

\section{Discussion}

The present study examined the effects of ice skating training during physical education classes on postural control. To the best of our knowledge, this study reports for the first time significantly enhanced balance performance in children when tested in postural transfer tasks.

The finding that gains in balance performance can be induced by alternative forms of balance training is well in line with observations made in adults and elderly people. It was, for example, shown that skiing (Lauber et al., 2011) and in-line skating (Taube et al., 2010) in elderly people are adequate means to improve postural stability. The same effect was observed when young adults (students) participated in slackline training (Keller et al., 2012; Pfusterschmied et al., 2013). Thus, it is possible to observe an enhanced postural control after

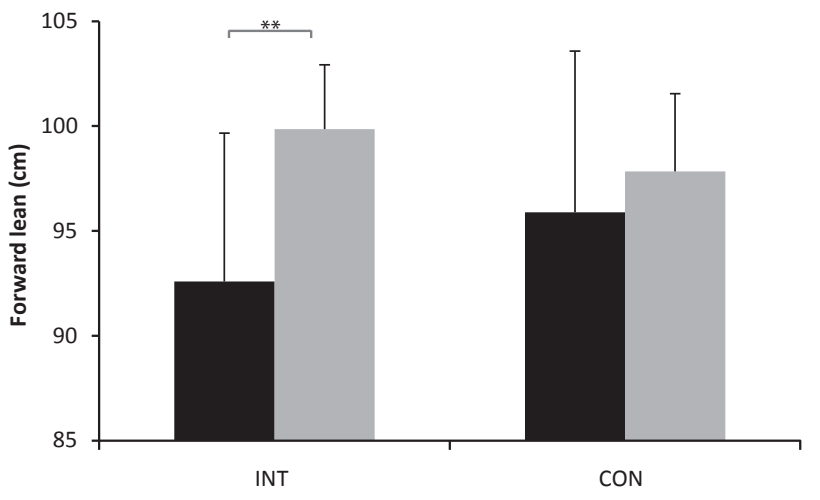

Fig. 3. This figure displays the group-specific changes in the functional reach test. Significant differences were found between the control (CON) and intervention (INT) groups over time with highly significant improved performance in the INT group and an unaltered performance in the $\mathrm{CON}(* * P<0.01)$.

several weeks of alternative balance training both when tested on the training device itself, but also when tested in a postural transfer test. The important finding of the present study is that this is also possible in children. To date, only one study reported enhanced postural control on the slackline after training this task for several weeks (Donath et al., 2013). However, the authors did not find any significant time $\times$ group interactions between the control and the training group when balance was tested in a transfer task. This is in line with the observations made by Granacher et al. (2011b) after conventional balance training in children showing non-significant adaptations in postural control. The present study, therefore, reports for the first time an improved balance performance in children when tested in untrained transfer tasks. This is especially relevant for the avoidance of falls as it is the aim to prepare children to counteract non-trained postural disturbances.

The present training regimen was chosen for its appealing character as it was previously shown that conventional balance training was not effective to improve significantly balance performance in children (Granacher et al., 2011b). As classical balance training interventions improved postural control in young adults (Gruber \& Gollhofer, 2004), middle-aged people (Granacher et al., 2011c), and old subjects (Granacher et al., 2010c), Granacher et al. (2011b) assumed that classical balance training might be relatively unattractive for children and, therefore, children might not have been intrinsically motivated to perform the exercises with high effort. This assumption was based on the observation that children only participated vigorously in the training when coaches were standing right beside them. Thus, ice skating may have counteracted this potential problem, especially as we constantly increased the task difficulty. Based on the energization theory of Brehm and Self (1989), stating that an adequate level of task difficulty is associated with increased motivation, one could argue that motivation was held constant throughout the training. 
Another factor that might explain why we found significant improvements in postural transfer tasks, although other studies did not (Granacher et al., 2011b; Donath et al., 2013), is the age of the subjects. The average age in our study was around 13 years, whereas younger subjects participated in the abovementioned studies (Donath et al., 2013: around 10 years; Granacher et al., 2011b: 6-7 years). One may argue that the maturity of the subjects may play an essential role as it is well established that young children use different postural control strategies to those of adults (Riach \& Hayes, 1987). Moreover, adult-like balance control does not appear before the age of 7 years (Riach \& Hayes, 1987). Thus, our findings are not directly comparable with those previous studies (Granacher et al., 2011b; Donath et al., 2013) because of age differences. Subjects in the current study represent the oldest cohort in comparison with the other studies and, therefore, it may be assumed that they already used an adult-like postural control.

\section{Considerations about the chosen posturography}

It has been previously discussed that easy balance tests like bipedal stance on a solid base of support can be non-adequate means when testing for functional relevant improvements in postural control (Taube et al., 2010). This assumption was supported by data from studies investigating the effect of Parkinson disease on human balance. For example, Smithson et al. (1998) were not able to describe any functional constraints in postural control when patients were tested in easy static balance tasks. The same authors, however, were able to describe significant impairments of postural stability when patients were tested in more challenging tasks. These observations were supported by further studies showing that parkinsonian patients can even show "better" postural control than healthy controls when tested in static balance tests but show serious constraints when the demands of the postural tasks are increased (Horak et al., 1992; Schieppati et al., 1994). Based on these findings, we decided to use the Posturomed in order to have a device capable of recording dynamic postural control with different levels of difficulty: a more easy condition (unperturbed upright stance) and a more challenging con- dition (lateral perturbation task). With this kind of setup we could ensure that all subjects were sufficiently challenged to keep their balance. Nevertheless, it would have been interesting to compare these results with results obtained with other kinetic or dynamic devices (like for instance reported in Taube et al., 2010). Unfortunately, we were not able to measure these data because of time constraints.

\section{Perspectives}

In summary, ice skating can be recommended for the promotion of balance in children aged 13 years. We acknowledge, however, that this study has some limitations that warrant discussion. For example, there is no reliable test to measure balance performance while ice skating. Therefore, we measured postural control only in transfer tasks. Unfortunately, time constraints due to fixed scholastic time tables prevented testing for possible side effects of balance training such as enhanced jump height (e.g., Taube et al., 2007b) or increased rate of force development (e.g., Gruber \& Gollhofer, 2004). Furthermore, we cannot identify the underlying neuromuscular mechanisms responsible for the observed effects. Upcoming studies should investigate supraspinal adaptations using imaging or stimulation methods as previous studies were able to correlate behavioral and physiological adaptations in response to balance training (Taube et al., 2007a; Taubert et al., 2010).

Despite the occurrence of some falls during the intervention period, no injury occurred that forced a participant to stop a training session or that disabled a participant to participate in the subsequent session. Therefore, ice skating can be recommended as a safe training method in case protective equipment is worn by all participants and the difficulty level is adapted to the average skill level of the group.

Key words: Postural control, balance training, children.

\section{Funding}

This project was supported by the Federal Office of Sport (BASPO, Magglingen, Switzerland).

\section{References}

Baudry S, Duchateau J. Age-related influence of vision and proprioception on Ia presynaptic inhibition in soleus muscle during upright stance. J Physiol 2012: 590: 5541-5554.

Boeer J, Mueller O, Krauss I, Haupt G, Horstmann T. [Reliability of a measurement technique to characterise standing properties and to quantify balance capabilities of healthy subjects on an unstable oscillatory platform (Posturomed)]. Sportverletz Sportschaden 2010: 24: 40-45.

Bös K, Oberger J, Lämmle L, Opper E, Romahn N, Tittlbach S, Wagner M, Woll A, Worth A. Motorische Leistungsfähigkeit von Kindern. In: Schmidt W, Zimmer R, Völker K, eds. Zweiter Deutscher Kinder- und Jugendsportbericht Schwerpunkt: Kindheit. Schorndorf: Hofmann Verlag, 2008: 136-157.
Brehm JW, Self EA. The intensity of motivation. Annu Rev Psychol 1989: 40: 109-131.

De Oreo K, Keogh J. Performance of fundamental motor tasks. In: Corbin $\mathrm{CB}$, ed. A textbook of motor development. Dubuque: Brown, 1980.

Donath L, Roth R, Rueegge A, Groppa M, Zahner L, Faude O. Effects of slackline training on balance, jump performance $\&$ muscle activity in 
young children. Int J Sports Med 2013: 34: 1093-1098.

Duncan PW, Weiner DK, Chandler J, Studenski S. Functional reach: a new clinical measure of balance. J Gerontol 1990: 45: M192-M197.

Granacher U, Gollhofer A, Kriemler S. Effects of balance training on postural sway, leg extensor strength, and jumping height in adolescents. Res Q Exerc Sport 2010a: 81: 245-251.

Granacher U, Iten N, Roth R, Gollhofer A. Slackline training for balance and strength promotion. Int J Sports Med 2010b: 31: 717-723.

Granacher U, Muehlbauer T, Bridenbaugh S, Bleiker E, Wehrle A, Kressig RW. Balance training and multi-task performance in seniors. Int $\mathrm{J}$ Sports Med 2010c: 31: 353-358.

Granacher U, Muehlbauer T, Gollhofer A, Kressig RW, Zahner L. An intergenerational approach in the promotion of balance and strength for fall prevention - a mini-review. Gerontology 2011a: 57: 304-315.

Granacher U, Muehlbauer T, Maestrini L, Zahner L, Gollhofer A. Can balance training promote balance and strength in prepubertal children? J Strength Cond Res 2011b: 25: 1759-1766.

Granacher U, Wick C, Rueck N, Esposito C, Roth R, Zahner L. Promoting balance and strength in the middle-aged workforce. Int J Sports Med 2011c: 32: 35-44.

Gruber M, Gollhofer A. Impact of sensorimotor training on the rate of force development and neural activation. Eur J Appl Physiol 2004: 92: 98-105.

Harriss DJ, Atkinson G. Update - ethical standards in sport and exercise science research. Int J Sports Med 2011: 32: 819-821.

Horak FB, Nutt JG, Nashner LM. Postural inflexibility in parkinsonian subjects. J Neurol Sci 1992: 111: 46-58.

Jurimae T, Volbekiene V, Jurimae J, Tomkinson GR. Changes in Eurofit test performance of Estonian and Lithuanian children and adolescents (1992-2002). Med Sport Sci 2007: 50: 129-142.

Kahl H, Dortschy R, Ellsasser G. [Injuries among children and adolescents (1-17 years) and implementation of safety measures. Results of the nationwide German Health Interview and Examination Survey for Children and Adolescents (KiGGS)].

Bundesgesundheitsblatt

Gesundheitsforschung

Gesundheitsschutz 2007: 50: 718-727.

Keller M, Pfusterschmied J, Buchecker M, Muller E, Taube W. Improved postural control after slackline training is accompanied by reduced H-reflexes. Scand J Med Sci Sports 2012: 22: 471-477.

Lauber B, Keller M, Gollhofer A, Muller E, Taube W. Spinal reflex plasticity in response to alpine skiing in the elderly. Scand J Med Sci Sports 2011: 21 (Suppl. 1): 62-68.

Ledebt A, Becher J, Kapper J, Rozendaalr RM, Bakker R, Leenders IC, Savelsbergh GJ. Balance training with visual feedback in children with hemiplegic cerebral palsy: effect on stance and gait. Motor Control 2005: 9: 459-468.

Mathers C, Penm R. Health system costs of injury, poisoning, and musculoskeletal disorders in Australia 1993-94. Canberra, Australia: Australian Institute of Health and Welfare, 1999.

Mombarg R, Jelsma D, Hartman E. Effect of Wii-intervention on balance of children with poor motor performance. Res Dev Disabil 2013: 34: 2996-3003.

Muller O, Gunther M, Krauss I,

Horstmann T. [Physical characterization of the therapeutic device Posturomed as a measuring device-presentation of a procedure to characterize balancing ability]. Biomed Tech (Berl) 2004: 49: 56-60.

Owens PL, Zodet MW, Berdahl T, Dougherty D, McCormick MC, Simpson LA. Annual report on health care for children and youth in the United States: focus on injury-related emergency department utilization and expenditures. Ambul Pediatr 2008: 8: 219-240.

Pfusterschmied J, Buchecker M, Keller M, Wagner H, Taube W, Muller E. Supervised slackline training improves postural stability. Eur J Sport Sci 2013: 13: 49-57.
Riach CL, Hayes KC. Maturation of postural sway in young children. Dev Med Child Neurol 1987: 29: 650-658.

Ruhe A, Fejer R, Walker B. The test-retest reliability of centre of pressure measures in bipedal static task conditions - a systematic review of the literature. Gait Posture 2010: 32: 436-445.

Schieppati M, Hugon M, Grasso M, Nardone A, Galante M. The limits of equilibrium in young and elderly normal subjects and in parkinsonians. Electroencephalogr Clin Neurophysiol 1994: 93: 286-298.

Smithson F, Morris ME, Iansek R. Performance on clinical tests of balance in Parkinson's disease. Phys Ther 1998: 78: 577-592.

Stevens JA, Corso PS, Finkelstein EA, Miller TR. The costs of fatal and non-fatal falls among older adults. Inj Prev 2006: 12: 290-295.

Taube W, Bracht D, Besemer C, Gollhofer A. The effect of inline skating on postural control in elderly people. Dtsch Z Sportmed 2010: 61: 45-51.

Taube W, Gruber M, Beck S, Faist M, Gollhofer A, Schubert M. Cortical and spinal adaptations induced by balance training: correlation between stance stability and corticospinal activation. Acta Physiol (Oxf) 2007a: 189: 347-358.

Taube W, Gruber M, Gollhofer A. Spinal and supraspinal adaptations associated with balance training and their functional relevance. Acta Physiol (Oxf) 2008: 193: 101-116.

Taube W, Kullmann N, Leukel C, Kurz O, Amtage F, Gollhofer A. Differential reflex adaptations following sensorimotor and strength training in young elite athletes. Int J Sports Med 2007b: 28: 999-1005.

Taubert M, Draganski B, Anwander A, Muller K, Horstmann A, Villringer A, Ragert P. Dynamic properties of human brain structure: learning-related changes in cortical areas and associated fiber connections. J Neurosci 2010: 30: 11670-11677. 